\title{
Potential Adverse Drug Reactions of Chloroquine in the Treatment of COVID-19
}

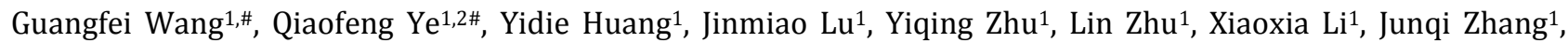
Ziwei $\mathrm{Li}^{1}$, Jianger $\mathrm{Lan}^{1}$ and Zhiping $\mathrm{Li}^{1}{ }^{*}$

${ }_{1}^{1}$ Department of Clinical Pharmacy, National Children's Medical Center, Children's Hospital of Fudan University, Shanghai, China

School of Pharmacy, Fudan University, Shanghai, China

\# Co-first authors: Guangfei Wang and Qiaofeng Ye contributed equally to this work.

* Corresponding author: Zhiping Li, Department of Clinical Pharmacy, National Children's Medical Center, Children's Hospital of Fudan University, Shanghai, China. Email: Zhiping Li, zpli@fudan.edu.cn

Received 2020 August 11; Revised 2020 September 26; Accepted 2020 0ctober 30.

\begin{abstract}
Context: Since December 2019, the coronavirus disease 2019 (COVID-19) has broken out in Wuhan, Hubei Province, China. Due to the highly pathogenic and infectious characteristics, COVID-19 spread across China and later globally and became a severe pandemic. To date, there have been no efficacious specialized drugs to treat COVID-19. The China-issued Diagnosis and Treatment of Pneumonia Caused by Novel Coronavirus (Trial version 6) added the chloroquine phosphate to the antiviral treatment protocol for infected adults.

Evidence Acquisition: In this review, government documents and authoritative guidelines on COVID-19 were collected from the official website of organizations related to health and medicine. Research articles related to chloroquine and its application for COVID-19 treatment were searched and acquired from the PubMed platform. Facts and data related to the use of chloroquine were summarized in several parts.

Results: Recently, there has been an increase in research on the use of chloroquine for the treatment of COVID-19. This drug is utilized as an antimalarial and antiviral medication. There are some concerns and cautions on the clinical application of chloroquine, about which clinicians should be informed during this global pandemic. The present review summarized data on the mechanism of action, drug-drug interaction (DDI), and adverse drug reaction (ADR) of chloroquine and pharmaceutical care for special patients in order to provide a reference for the rational use of this drug in COVID-19 patients.

Conclusion: Currently, there is mixed evidence on the efficacy of chloroquine in the treatment of COVID-19. Potential DDIs and ADRs, as well as pharmaceutical care, for special patients should be considered in fighting against the pandemic.

Keywords: Adverse drug reactions, Chloroquine, COVID-19
\end{abstract}

\section{Context}

In December 2019, the first case of coronavirus disease 2019 (COVID-19) was reported in Wuhan, China. The disease quickly spread to the other regions of this country and later became a global pandemic. The World Health Organization (WHO) has announced this disease as a public health emergency of international concern. Accordingly, the effective prevention and treatment of COVID-19 have become a major problem. The virus causing COVID-19 was originally called 2019 Novel Coronavirus (2019-nCoV). Later, research found that the virus is genetically related to the coronavirus that accounted for severe acute respiratory syndrome (SARS) in 2003; accordingly, it was named SARS-CoV-2. There is a similarity of $79.5 \%$ in genome sequence between the two viruses. Angiotensin-converting enzyme 2 (ACE2) is the receptor of SARS-CoV-2 on cell surface, which interacts with the spike glycoprotein (S protein) of the virus to allow entrance $(1,2)$.

Currently, most of the drugs administered for the treatment of COVID-19 are among those used for SARS treatment (3). Research has found that chloroquine can effectively inhibit the infection and spread of SARS-CoV (4). The "Diagnosis and Treatment of Pneumonia Caused by Novel Coronavirus (Trial version 6)" issued by the National Health Commission of China added chloroquine phosphate to the antiviral treatment protocol for infected adults (5). On February 21 ${ }^{\text {st }}, 2020$, the Health Commission of Hubei issued the "Notice on Close Monitoring for Adverse Drug Reactions of Chloroquine". According to the report from the Wuhan Institute of Virology, Chinese Academy of Sciences, the lethal dose of chloroquine phosphate in adults is 2-4 g. Therefore, designated institutions are required to closely monitor adverse drug reactions (ADRs) when using this drug.

On February 28 $8^{\text {th }}, 2020$, the National Health Commission of China issued the "Notice on Adjusting the Dosing Regimen of Chloroquine Phosphate", declaring chloroquine phosphate to be appropriate for COVID-19 patients at the age of 18-65 years. They stated that the dose should be adjusted according to patient's weight and also announced the absolute and relative contraindications and the caveats (6). The "Diagnosis and Treatment of Pneumonia Caused by Novel Coronavirus (Trial version 7)" recommends the 
adjusted dose and clearly points out that close attention should be paid to ADRs, contraindications, special population, and drug-drug interactions (DDIs) during the use of chloroquine phosphate (7). Pharmacists, as the first accountable agents and also the final guards of the safe use of drugs, should have a good command of knowledge regarding the mechanism of action, DDIs, and ADRs of medications. This review intended to provide a reference for clinicians to promote pharmaceutical care so as to reduce controllable ADRs of chloroquine and enhance the proper use of the drug in COVID-19 patients.

\section{Evidence Acquisition}

\subsection{Data sources}

Government documents and authoritative guidelines on COVID-19 were collected from the official website of organizations related to health and medicine, such as the WHO, National Institute of Health (NIH), and National Health Commission of the People's Republic of China. In addition, research articles, reviews, or case reports related to chloroquine and its application for COVID-19 treatment were searched and obtained from the PubMed platform, which is an open authoritative database with more than 30 million citations for medical literature. Electronic and paper books, such as the 'Pediatric \& Neonatal Dosage Handbook', were also referred for dosage recommendation and other relevant information on the use of chloroquine.

\subsection{Search strategy}

The data were searched in the PubMed database using the following keywords: "COVID-19" OR "coronavirus" OR "novel coronavirus" OR "2019nCov" OR "NCP" OR "novel coronavirus pneumonia" AND "chloroquine" OR "hydroxychloroquine" OR "CQ" OR "HCQ" OR "antimalarial". All articles found in the initial search were screened based on their titles and abstracts. The articles containing information related to the development of chloroquine, clinical application of chloroquine, efficacy of chloroquine in COVID-19 treatment, dosage recommendations of the drug, adverse drug reactions or adverse drug events related to chloroquine, potential DDI, and chloroquine application in special populations were included in the study. However, irrelevant articles were excluded from the review process. Facts and data related to the use of chloroquine were collected and summarized in several parts.

\section{Results}

\subsection{Chloroquine in clinical application}

Chloroquine is a 4-aminoquinoline medication used to treat and prevent malaria. It is also used for amebiasis that is occurring outside of the intestines, rheumatoid arthritis, and systemic lupus erythematosus. In 1934, chloroquine and 3methylquinoline were synthesized successfully. Affected by the toxicity of chloroquine, people did not pay much attention to the application of the medication until World War II. Thereafter, the American government began to sponsor the clinical trials of chloroquine as an antimalarial agent. The results of the trials showed the appropriateness of chloroquine as an antimalarial drug, which led to its approval in 1947. During the 1960s-1970s, chloroquine became the most pervasively used and synthesized antimalarial agent (8). Upon more investigations by scientists, chloroquine was found to have not only anti-malarial activity but also immuneregulating and antiviral effects. Since then, this medication has been often used in autoimmune diseases, such as rheumatoid arthritis and systemic lupus erythematosus (SLE) (9). The most common forms of chloroquine compounds are chloroquine phosphate, chloroquine sulfate, and chloroquine hydrochloride, the molecular structures of which are shown in Figure 1.<smiles>CCN(CC)CCCC(C)Nc1ccnc2cc(Cl)ccc12</smiles><smiles>CCN(CC)CCCC(C)Nc1ccnc2cc(Cl)ccc12</smiles><smiles>CCN(CC)CCCC(C)Nc1ccnc2cc(Cl)ccc12</smiles><smiles>CCN(CC)CCCC(C)Nc1ccnc2cc(Cl)ccc12</smiles>

Figure 1. Structures of chloroquine 


\subsection{Current situation of chloroquine against coronavirus}

Coronavirus is an enveloped, positive-sense, single-stranded RNA virus, pervasively existing in nature. Based on the evidence, the genome sequence of SARS-CoV-2 is $96 \%$ consistent with that of RaTG13 coronavirus in Rhinolophus affinis isolated from Yunnan province, China. Therefore, it is conjectured that the natural host of SARS-CoV-2 is bat (1). To date, coronavirus has shown potential lethal effects on humans. The known coronaviruses that can cause epidemic include SARS-CoV that broke out in 2003 with a case-fatality rate of around $10 \%$, Middle-East respiratory syndrome coronavirus (MERS-CoV) first emerging in 2012 with a case-fatality rate of almost $30 \%$, and SARS-CoV-2 first reported in December 2019 , without an accurate case-fatality rate since the pandemic has not got over yet. Bioinformatic analysis found that the structure of the $S$ protein of SARS-CoV-2 is similar to that of SARS-CoV, which can also combine with ACE2 receptor on the host cell surface, thereby entering the epithelial cells of the host $(1,10)$.

The possible mechanisms of the antiviral activity of chloroquine include: (I) interference of chloroquine with the glycosylation of the end of ACE2 receptor and inhibition of viral invasion (3), (II) accumulation of chloroquine, as an alkaline compound, in the acidic cell organelles (e.g., nucleus, the vesicles of Golgi apparatus, and lysosome), which can prevent the viral infection via blocking the $\mathrm{pH}$ dependent endocytosis $(9,11)$, (III) immuneregulation effect of chloroquine, which can prevent the production of TNF- $\alpha$, IL-6, and IFN- $\gamma$ and decrease immunopathogenic impairment (currently used in the treatment of autoimmune diseases, such as rheumatoid arthritis and SLE) (9). In the middle and late stages of SARS-CoV-2 infection, the concentrations of TNF- $\alpha$ and IL- 6 are associated with the severity of diseases. Therefore, the antiinflammatory effect of chloroquine may help decrease the immunopathogenic damage.

In terms of anti-coronavirus efficacy, in 2004, a group of Belgian researchers (12), performing in vitro cell experiment, reported that chloroquine phosphate showed antiviral activity against SARS$\mathrm{CoV}$ infected cells, with the $\mathrm{IC}_{50}$ value of $8.8 \pm 1.2 \mu \mathrm{M}$, $\mathrm{CC}_{50}$ value of $261.3 \pm 14.5 \mu \mathrm{M}$, and selective index (SI) of 30 , which is close to the concentration treating acute malaria. In 2014, Dutch researchers (13) found that chloroquine could inhibit the replication of the virus in SARS-CoV-infected Vero E6 cells and MERS-CoV-infected Huh8 cells. In the mentioned study, the effect was reported to be dose-dependent with the $\mathrm{EC}_{50}$ values of $4.1 \pm 1.0$ and $3.0 \pm 1.1 \mu \mathrm{M}, \mathrm{CC}_{50}$ values of $>128$ and $58.1 \pm 1.1 \mu \mathrm{M}$, and SI values of $>31$ and 19.4 for the aforementioned cells, respectively. In December 2019, the results found in a cooperative study performed by the Wuhan Institute of Virology, Chinese Academy of Sciences,
Beijing Institute of Toxicology and Pharmacology, and Academy of Military Sciences demonstrated the efficacy of the low concentrations of remdesivir $\left(\mathrm{EC}_{50}=0.77 \mu \mathrm{M}, \quad \mathrm{CC}_{50}>100 \mu \mathrm{M}, \quad \mathrm{SI}>129.87\right)$ and chloroquine $\left(\mathrm{EC}_{50}=1.13 \mu \mathrm{M}, \mathrm{CC}_{50}>100 \mu \mathrm{M}, \mathrm{SI}>88.50\right)$ in blocking SARS-CoV-2 infection with high selectivity (4).

Recently, several clinical studies have been carried out rendering new findings. There is mixed evidence regarding the effect of chloroquine on COVID-19. In a parallel, double-blinded randomized clinical trial, the safety and efficacy of high dose $(600$ mg twice daily for 10 days) with low dose (450 mg twice daily on day 1 and once daily for 4 days) of chloroquine diphosphate were compared among 81 adult patients (14). The results showed that case fatality rate until day 13 in the high-dose group $(39.0 \%)$ was higher than that in the low-dose group (15.0\%). In addition, the high-dose group had more cases of QTc interval prolongation (18.9\%), compared to the low-dose group (11.1\%) (14). Based on the mentioned study, it is not recommended to take a high dosage of chloroquine for critically ill patients with COVID-19, especially old patients with underlying cardiac diseases. In a recent multicenter observational study performed in China, the data were indicative of the positive effect of chloroquine. In the mentioned research, the median time to achieve an undetectable viral RNA was shorter in the chloroquine group than in the non-chloroquine group (-6.0 days), and the duration of fever was shorter (15). In addition, no serious ADRs were reported in the aforementioned study (15). Nevertheless, it is worth noting that there was a significant difference between the experimental group treated with chloroquine and the control group without chloroquine in terms of the median time interval between the symptom onset and the treatment onset (15). Therefore, the self-limiting nature of the disease is probably the major factor playing a role in recovery.

An article from the American College of Physicians indicated the lack of evidence on using chloroquine for COVID-19 prophylaxis and treatment. In the mentioned study, it was argued that clinicians should not use chloroquine alone or in combination with azithromycin as prophylaxis for the general population or as a treatment for patients with COVID19 unless in the context of clinical trial with informed consent from the patients or their families (16). With a lot of debate around the efficacy and safety of chloroquine and the currently available evidence, the U.S. Food and Drug Administration (FDA) eventually revoked the Emergency Use Authorization for the emergency use of chloroquine to treat COVID-19 on June 15, 2020 (17). Meanwhile, the NIH COVID-19 Treatment Guidelines Panel recommends against the use of chloroquine for the treatment of COVID-19, except in a clinical trial (18). 


\subsection{Dosage of chloroquine}

Chloroquine phosphate is currently used as an experimental treatment for COVID-19 infected adults within the age of 18-65 years. In the Chinese guideline, for patients with a weight of $>50 \mathrm{~kg}$, it is recommended to take $500 \mathrm{mg}$ per dose, twice daily, 7 days for a course. However, for those weighing $\leq$ $50 \mathrm{~kg}$, the recommended dosing amount is $500 \mathrm{mg}$ per dose, twice daily for the first 2 days, followed by $500 \mathrm{mg}$ per dose, once daily for the next 5 days, with a course of 7 days in total (6). Regimens used in clinical trials and other guidelines vary from 250 to $1000 \mathrm{mg}$ per day for approximately 5 days (19). To date, there has been no recommendation for pediatric patients with COVID-19. The dosage can be referred to the dosage regimen for treating extraintestinal amoebiasis and liver abscess in pediatric patients.

According to the 'Pediatric \& Neonatal Dosage Handbook' by Lexicomp (20), chloroquine phosphate can be used to treat pediatric extraintestinal amoebiasis and liver abscess, taken with metronidazole or tinidazole. The oral dosage recommended is $16.6 \mathrm{mg} / \mathrm{kg} /$ dose, once daily, at a maximal dose of $1000 \mathrm{mg} /$ dose, followed by its replacement with paromomycin or iodoquinoline after 21 days. The oral dosage used to prevent malaria in children is $8.4 \mathrm{mg} / \mathrm{kg} /$ dose, once a week, at a maximal dose of $500 \mathrm{mg} /$ dose, started 1-2 weeks before exposure and continued for 4 weeks after leaving the endemic region. The oral dosage used to treat malaria in children is $16.6 \mathrm{mg} / \mathrm{kg} /$ dose at a maximal dose of $1000 \mathrm{mg} /$ dose for loading dose, followed by $8.3 \mathrm{mg} / \mathrm{kg} /$ dose taken 6,24 , and $48 \mathrm{~h}$ after the administration of the loading dose.

\subsection{Drug-drug interactions}

For COVID-19 patients with other underlying diseases, it is common to combine this medication with other medications. Multiple drug combinations can increase the risk of ADRs and even have lethal effect. As the first responsible agents for prescription review, pharmacists have to pay attention to potential DDIs and prevent the incidence of adverse drug events. Chloroquine is mainly metabolized by liver enzymes, namely CYP3A5 and CYP2D6, and can prolong the QT interval. Regarding this, caution should be exercised when prescribing this medication along with the drugs that can cause arrhythmia or inhibit the activity of CYP2D6 and CYP3A4 (20).

Patients, who have to take such medications as digitalis, quinolones (e.g., levofloxacin, moxifloxacin, and sparfloxacin), macrolides (e.g., azithromycin, erythromycin, and clarithromycin), phenylbutazone, heparin, penicillamine, amiodarone, bepridil, domperidone, droperidol, haloperidol, astemizole, posaconazole, methadone, procainamide, hydrochlorothiazide, cisapride, indapamide, chlorpromazine, streptomycin, ammonium chloride, ondansetron, apomorphine, octreotide, monoamine oxidase inhibitor, and triamcinolone should be prohibited from taking chloroquine (6).

In addition, the combined use of chloroquine with other drugs lowering blood glucose level will increase the risk of hypoglycemia. Cimetidine can inhibit the metabolism of chloroquine, leading to an increase in the plasma concentration of chloroquine and symptoms of intoxication, such as agitation, onset of epilepsy, and cardiac arrest. When taken with cyclosporin, chloroquine can increase the concentration of cyclosporin, inducing toxic reactions related to cyclosporin, requiring therapeutic drug monitoring (21). The common DDIs are summarized in Table $1(20,21)$.

\subsection{Common adverse drug reactions}

Chloroquine phosphate tablets are absorbed after oral administration and distributed extensively in tissues by accumulating in the liver, spleen, kidney, lung, and tissues, containing melanin (e.g., retina and inner ear) (22). Pharmacokinetic research has found that the half-life of chloroquine in plasma can be as long as 2.5-10 days, and even longer in tissues (22).

\begin{tabular}{|c|c|}
\hline Effect of DDIs & Drugs that cause the DDIs \\
\hline $\begin{array}{l}\text { Increased efficacy/ } \\
\text { toxicity of the combined } \\
\text { drug }\end{array}$ & $\begin{array}{l}\text { Antipsychotics (phenothiazines), } \beta \text {-blockers, cardiac glycoside, dapsone (systemic or local apply), } \\
\text { hypoglycemia-related drugs, lumefantrine, perhexiline, prilocaine, primaquine, mefloquine, drugs related } \\
\text { to the risk of QT interval prolongation (high risk or medium risk), sodium nitrite, and cyclosporin }\end{array}$ \\
\hline $\begin{array}{l}\text { Increased efficacy/ } \\
\text { toxicity of chloroquine }\end{array}$ & $\begin{array}{l}\text { Abiraterone acetate, androgen, drugs for diabetic mellitus (traditional Chinese medicine or western } \\
\text { drugs), aprepitant, artemether, asunaprevir, cimetidine, conivaptan, CYP2D6 inhibitors (potent or } \\
\text { medium), CYP3A4 inhibitors (potent or medium), dapsone (systemic), fosaprepitant, fusidic acid } \\
\text { (systemic), hydroxychloroquine, mefloquine, idelalisib, imatinib, macimorelin, mifepristone, monoamine } \\
\text { oxidase inhibitors, netupitant, nitric oxide, palbociclib, panobinostat, interferon } \alpha \text {-2b, pegvisomant, } \\
\text { perhexiline, probucol, promazine, prothionamide, drugs related to the risk of QT interval prolongation } \\
\text { (uncertain risk and risk under revision), quinolone, salicylate, selective serotonin reuptake inhibitors, } \\
\text { simeprevir, stiripentol, tamoxifen, tetracaine hydrochloride (local), vinflunine, xipamide, domperidone, } \\
\text { and streptomycin }\end{array}$ \\
\hline $\begin{array}{l}\text { Decreased efficacy of the } \\
\text { combined drug }\end{array}$ & $\alpha$-Galactosidase, $\beta$-galactosidase, ampicillin, bacampicillin, praziquantel, and rabies vaccine \\
\hline $\begin{array}{l}\text { Decreased efficacy of the } \\
\text { chloroquine }\end{array}$ & $\begin{array}{l}\text { Antacid (magnesium aluminum carbonate), bosentan, CYP3A4 inducers (potent, medium), dabrafenib, } \\
\text { deferasirox, enzalutamide, mituotan, peginterferon } \alpha-2 b \text {, pitolisant, bortezomib, and tocilizumab }\end{array}$ \\
\hline
\end{tabular}


Common ADRs include dizziness, headache, blurred vision, loss of appetite, nausea, vomiting, abdominal pain, diarrhea, and rash, most of which are mild symptoms and will disappear after stopping taking the drug. In addition, severe ADRs and DDIs, such as psychiatric symptoms, severe extrapyramidal symptoms, cardiac arrest, and ocular symptoms, should be timely noticed and managed. In case of the emergence of intolerable toxic side effects, it is required to stop medicine administration and report the ADR strictly according to the "Regulations on ADR Reporting and Monitoring Management".

According to the "Pediatric \& Neonatal Dosage Handbook" by Lexicomp (20) and relative literature, cardiac toxicity is the most severe ADR of chloroquine phosphate. Furthermore, ocular and hearing impairments are the major ADRs of chloroquine phosphate. These ADRs are summarized as follows:

(I) Cardiac toxicity: cardiac toxicity is the most severe ADR, which can inhibit sinoatrial node, arrhythmia (QT interval prolongation), and shock and even lead to Adams-Stokes syndrome or death under severe conditions (23-25). According to a meta-analysis, about $10 \%$ of COVID-19 patients treated with chloroquine or hydroxychloroquine developed QT prolongation (19). The general risk factors for QT prolongation and torsades de pointes when treated with the drug include congenital long QT syndrome (26), combination with multiple QTprolonging medications (27), and female sex (26). In addition, illness-related risk factors, including hypokalemia (28), hypomagnesemia (28), sepsis (27), myocardial injury (27), ischemia (27), heart failure (27), renal impairment (27), bradycardia (28), and recent conversion from atrial fibrillation (26), can increase the possibility of the cardiac toxicity of the drug.

(II) Effect on the eyes $(25,29,30)$ : the ADR to the eyes is mainly the retinopathy. Orally taken chloroquine can accumulate in tissues. Long-term usage can lead to macular degeneration and has an impact on vision. The high-risk factors causing retinopathy include the duration of administration, daily maximal dose, and cumulative dosage. Based on the evidence, the incidence of the retinal toxicity of chloroquine is higher than that of hydroxychloroquine (31). Screening for patients' vision problems should be executed when using chloroquine.

(III) Effect on hearing (24): it was reported that the long-term use of 4-aminoquinoline could cause tinnitus, dizziness, and nervous deafness because of its affinity to the melanocytes of the stria vascularis and ampulla of semicircular canals. Patients with hearing impairment should use this medication with caution.

(IV) Influence on the hematological system (24): ADRs related to hematological systems are relatively few. Some of them include hemolysis, aplastic anemia, reversible agranulocytosis, and thrombocytopenia. In a study, some of the patients treated with chloroquine presented hematological ADRs $(n=3)$, aplastic anemia $(n=1)$, aplastic anemia followed by acute myeloid leukemia $(n=1)$, and pancytopenia and aplastic anemia $(n=1)$ after taking chloroquine for 3 weeks (32).

(V) Effect on glucose metabolism: chloroquine can reportedly induce hypoglycemia, happening in patients with or without antidiabetics (33). It is suggested that clinicians should closely monitor blood glucose level and hypoglycemic symptoms.

(VI) Influence on skin: macular papule is the most common dermal ADR. It usually appears in 1-3 weeks after medication. Since this medication is dosedependent, there is no need to stop taking the drug (34). Other rare ADRs include hair loss, exfoliative dermatitis, and cutaneous vasculitis $(24,34)$.

(VII) Other ADRs: the other ADRs include neuromuscular pain, drug-induced neuropsychosis, and dysmyotonia $(24,35,36)$.

\subsection{Pharmaceutical care for special populations}

Healthcare workers and researchers are exploring the best protocol for COVID-19 treatment. Chloroquine phosphate is experimentally used for adult patients with 18-65 years of age. The contraindications and relative contraindications of chloroquine phosphate are $(6,20)$ pregnancy, allergy to 4-aminoquinoline, arrhythmia (conduction blockade), chronic cardiac diseases, psychosis, dermal lesions (rash, dermatitis, and psoriasis. Chloroquine can induce acute toxic hepatitis in patients with porphyria cutanea tarda and glucose-6phosphate dehydrogenase deficiency; furthermore, it may cause hemolysis.

All types of populations are susceptible to SARSCoV-2. In addition to attention to dosage, DDIs, and ADRs, pharmaceutical care for special populations should never be ignored. In pediatric population, chloroquine can be used as prevention or treatment for malaria in infants, children, and teenagers (20). When daily dose is relatively large or duration is over 5 years, attention should be paid to possible retinal toxicity. The dosage used to treat children with COVID-19 can be referred to the dosage used to prevent and treat malaria in children. With regard to pregnant women, chloroquine can cross the placental barrier. There is insufficient evidence about its effect on fetus, and research conclusions are inconsistent. It is clearly pointed out on the package insert of chloroquine that it is prohibited to put pregnant women on this drug. However, in clinical situations, doctors can prescribe this medication for pregnant women after weighing the risks and benefits (20).

Wittes et al. (24) conducted a meta-analysis on the fetal deformity of pregnant women treated with chloroquine and concluded that chloroquine might be 
the cause. The results of another observational study by Wolfe et al. (37) revealed no significant difference between chloroquine-exposure and non-exposure groups in terms of the incidence of fetal deformity. In a meta-analysis carried out by Ruiz-Irastorza et al. (31), the authors reported that chloroquine and hydroxychloroquine could be used for pregnant women and that hydroxychloroquine was at a higher level on the ladder of safety evidence. Based on the current evidence, the influence of chloroquine on fetus cannot be completely ruled out; therefore, pregnant women should go through sufficient clinical evaluation before being put on this medicine.

In terms of breastfeeding women, there is controversial evidence on the safety of using chloroquine. In 2002, the American Academy of Pediatrics (38) updated the statement on drugs or chemicals that can be secreted to lactic milk, with the view that chloroquine can be used in lactation period. However, according to the package insert, this drug should be cautiously used for breastfeeding women. Therefore, long-term or high-dose usage of this medication in breastfeeding women should be based on weighing the associated benefits and risks.

With regard to elderly patients, there is no guideline for using this drug for this population. Considering that chloroquine is mainly excreted in the kidney, the dose should be adjusted for old people without normal renal function. For the patients with the risk of QT interval prolongation $(19,39)$, it is clearly stated in the package insert of chloroquine phosphate that this drug can block the sinoatrial node, causing arrhythmia, shock, and even AdamsStrokes syndrome or death in severe cases. Patients with cardiac disease history should use this medication with caution. Generally, patients with COVID-19 are at a greater risk of QT interval prolongation given the high possibility of myocardial injury and heart failure (40). Therefore, baseline and repeated measurement of electrocardiogram is recommended for these patients (41-43).

In patients with hepatic and renal function insufficiency $(22,44)$, chloroquine is mainly metabolized in the liver and accumulated in hepatic tissue. Additionally, for patients with insufficient hepatic function, especially those with hepatic cirrhosis, relative ADRs should be closely monitored. Under the condition of renal impairment, the time of excretion is often prolonged. There is no need to adjust the dosage, but for long-term users, it is recommended to lower its dose.

\section{Conclusion}

Currently, there is some clinical experience regarding chloroquine phosphate; however, the evidence is mixed on the efficacy of this medication in the treatment of COVID-19. Randomized clinical trials with a large sample size are needed to further clarify the efficacy and safety of chloroquine in treating COVID-19. Research on special populations is lacking, and more research evidence is needed to keep up with the progressive spread of the virus. Caution should be exercised since chloroquine has many ADRs, making it risky when overdosing. According to the national requirements of China, close attention should be paid to ADRs, contraindications, special populations, and DDIs. As part of the frontline fighting against the pandemic, pharmacists are the first responsible agents in prescription review and the final gatekeepers for the safe use of drugs. As a result, they should have a good command of knowledge regarding the mechanism of action, DDIs, ADRs, and pharmaceutical care for special populations using chloroquine to ensure a safe and effective pharmacological treatment. The achievement of information regarding chloroquine can inform the close collaboration of the whole healthcare team, promote the proper use of this drug, and fill the gaps in treating patients with COVID-19.

\section{Footnotes}

Conflict of Interests: The authors declare no conflict of interest.

Funding: This work was supported by the National Natural Science Foundation (No. 81874325), Scientific Research Project of Science and Technology Commission of Shanghai Municipality (No. 19DZ1910604/19XD1400900/18DZ1910604), and Key Innovative Team of Shanghai Top-Level University Capacity Building in Clinical Pharmacy and Regulatory Science at Shanghai Medical College, Fudan University (No. HJW-R-2019-66-19).

\section{References}

1. Zhou P, Yang XL, Wang XG, Hu B, Zhang L, Zhang W, et al. A pneumonia outbreak associated with a new coronavirus of probable bat origin. Nature. 2020;579(7798):270-3. doi: 10.1038/s41586-020-2012-7. [PubMed: 32015507].

2. Yan R, Zhang Y, Li Y, Xia L, Guo Y, Zhou Q. Structural basis for the recognition of SARS-CoV-2 by full-length human ACE2. Science. 2020;367(6485):1444-8. doi: 10.1126/science.abb 2762. [PubMed: 32132184].

3. Vincent MJ, Bergeron E, Benjannet S, Erickson BR, Rollin PE, Ksiazek TG, et al. Chloroquine is a potent inhibitor of SARS coronavirus infection and spread. Virol J. 2005;2:69. doi: 10.1186/1743-422X-2-69. [PubMed: 16115318].

4. Wang M, Cao R, Zhang L, Yang X, Liu J, Xu M, et al. Remdesivir and chloroquine effectively inhibit the recently emerged novel coronavirus (2019-nCoV) in vitro. Cell Res. 2020;30(3):269-71. doi: 10.1038/s41422-020-0282-0. [PubMed: 32020029].

5. Health Commission of People's Republic of China. Diagnosis and treatment of pneumonia caused by novel coronavirus. $6^{\text {th }}$ ed. China: NHCotPsRo; 2020.

6. Adjustment on the usage of chloroquine phosphate for treating 2019 coronavirus disease. National Health Commission and National Administrative Office of Chinese Tradition Medicine. Available at: URL: http://www.nhc.gov.cn/yzygj/s7653p/ 202002/0293d017621941f6b2a4890035243730.shtml; 2020.

7. Health Commission of People's Republic of China. Diagnosis 
and treatment of pneumonia caused by novel coronavirus. $7^{\text {th }}$ ed. China: NHCotPsRo; 2020.

8. Packard RM. The origins of antimalarial-drug resistance. $N$ Engl J Med. 2014;371(5):397-9. doi: 10.1056/NEJMp1403340. [PubMed: 25075832].

9. Savarino A, Boelaert JR, Cassone A, Majori G, Cauda R. Effects of chloroquine on viral infections: an old drug against today's diseases? Lancet Infect Dis. 2003;3(11):722-7. doi: 10.1016/s1473-3099(03)00806-5. [PubMed: 14592603].

10. Xu X, Chen P, Wang J, Feng J, Zhou H, Li X, et al. Evolution of the novel coronavirus from the ongoing Wuhan outbreak and modeling of its spike protein for risk of human transmission. Sci China Life Sci. 2020;63(3):457-60. doi: 10.1007/s11427020-1637-5. [PubMed: 32009228].

11. Yang ZY, Huang Y, Ganesh L, Leung K, Kong WP, Schwartz O, et al. pH-dependent entry of severe acute respiratory syndrome coronavirus is mediated by the spike glycoprotein and enhanced by dendritic cell transfer through DC-SIGN. J Virol. 2004;78(11):5642-50. doi: 10.1128/JVI.78.11.56425650.2004. [PubMed: 15140961].

12. Keyaerts E, Vijgen L, Maes P, Neyts J, Van Ranst M. In vitro inhibition of severe acute respiratory syndrome coronavirus by chloroquine. Biochem Biophys Res Commun. 2004; 323(1):264-8. DOI: 10.1016/j.bbrc.2004.08.085. [PubMed: $15351731]$.

13. de Wilde AH, Jochmans D, Posthuma CC, Zevenhoven-Dobbe JC, van Nieuwkoop S, Bestebroer TM, et al. Screening of an FDAapproved compound library identifies four small-molecule inhibitors of Middle East respiratory syndrome coronavirus replication in cell culture. Antimicrol Agents Chemother. 2014;58(8):4875-84. doi: 10.1128/AAC.03011-14. [PubMed: 24841269].

14. Borba MG, Val FF, Sampaio VS, Alexandre MA, Melo GC, Brito $\mathrm{M}$, et al. Effect of high vs low doses of chloroquine diphosphate as adjunctive therapy for patients hospitalized with severe acute respiratory syndrome coronavirus 2 (SARS-CoV-2) infection: a randomized clinical trial. JAMA Netw Open. 2020;3(4):e208857. doi: 10.1001/jamanetworkopen.2020.8857. [PubMed: 32339248].

15. Huang M, Li M, Xiao F, Pang P, Liang J, Tang T, et al. Preliminary evidence from a multicenter prospective observational study of the safety and efficacy of chloroquine for the treatment of COVID-19. Natl Sci Rev. 2020;7(9):1428-36. doi: 10.1093/nsr/nwaa113.

16. Qaseem A, Yost J, Etxeandia-Ikobaltzeta I, Miller MC, Abraham GM, Obley AJ, et al. Correction: should clinicians use chloroquine or hydroxychloroquine alone or in combination with azithromycin for the prophylaxis or treatment of COVID19? Ann Intern Med. 2020;173(2):166. doi: 10.7326/L20-0684. [PubMed: 32449881].

17. Coronavirus (COVID-19) update: FDA revokes emergency use authorization for chloroquine and hydroxychloroquine. US Food and Drug Administration. Available at: URL: https://www.fda.gov/news-events/press-announcements/ coronavirus-covid-19-update-fda-revokes-emergency-useauthorization-chloroquine-and?from=groupmessage\&isappin stalled $=0 ; 2020$.

18. National Institutes of Health. COVID-19 treatment guidelines panel. coronavirus disease 2019 (COVID-19) treatment guidelines. New York: National Institutes of Health; 2020.

19. Jankelson L, Karam G, Becker ML, Chinitz LA, Tsai MC. QT prolongation, torsades de pointes and sudden death with short courses of chloroquine or hydroxychloroquine as used in COVID-19: a systematic review. Heart Rhythm. 2020; 17(9):1472-9. doi: 10.1016/j.hrthm.2020.05.008. [PubMed: 32438018].

20. Taketomo CK, Hodding JH, Kraus DM. Pediatric \& neonatal dosage handbook: a comprehensive resource for all clinicians treating pediatric and neonatal patients. $25^{\text {th }}$ ed. Ohio: Lexicomp; 2018.

21. Schlossberg D, Samuel R. Antibiotics manual: a guide to commonly used antimicrobials. $2^{\text {nd }}$ ed. New Jersey: John Wiley \& Sons; 2017.

22. Ducharme J, Farinotti R. Clinical pharmacokinetics and metabolism of chloroquine. Focus on recent advancements. Clin Pharmacokinet. 1996;31(4):257-74. doi: 10.2165/ 00003088-199631040-00003. [ PubMed: 8896943].

23. Stas P, Faes D, Noyens P. Conduction disorder and QT prolongation secondary to long-term treatment with chloroquine. Int J Cardiol. 2008;127(2):e80-2. doi: 10.1016/j. ijcard.2007.04.055. [PubMed: 17590456].

24. Wittes R. Adverse reactions to chloroquine and amodiaquine as used for malaria prophylaxis: a review of the literature. Can Fam Physician. 1987;33:2644-9. [PubMed: 21264010].

25. Cervera A, Espinosa G, Font J, Ingelmo M. Cardiac toxicity secondary to long term treatment with chloroquine. Ann Rheum Dis. 2001;60(3):301. doi: 10.1136/ard.60.3.301. [PubMed: 11245140].

26. Roden DM. Drug-induced prolongation of the QT interval. $N$ Engl J Med. 2004;350(10):1013-22. doi: 10.1056/ NEJMra032426. [ PubMed: 14999113].

27. Tisdale JE. Drug-induced QT interval prolongation and torsades de pointes: role of the pharmacist in risk assessment, prevention and management. Can Pharm J. 2016;149(3):13952. doi: 10.1177/1715163516641136. [PubMed: 27212965].

28. Kannankeril P, Roden DM, Darbar D. Drug-induced long QT syndrome. Pharmacol Rev. 2010;62(4):760-81. doi: 10.1124/pr.110.003723. [PubMed: 21079043].

29. Michaelides M, Stover NB, Francis PJ, Weleber RG. Retinal toxicity associated with hydroxychloroquine and chloroquine: risk factors, screening, and progression despite cessation of therapy. Arch Ophthalmol. 2011;129(1):30-9. doi: 10.1001/archophthalmol.2010.321. [PubMed: 21220626].

30. Costedoat-Chalumeau N, Dunogué B, Leroux G, Morel N, Jallouli $M$, Le Guern V, et al. A Critical review of the effects of hydroxychloroquine and chloroquine on the eye. Clin Rev Allergy Immunol. 2015;49(3):317-26. doi: 10.1007/s12016015-8469-8. [PubMed: 25672591].

31. Ruiz-Irastorza G, Ramos-Casals M, Brito-Zeron P, Khamashta MA. Clinical efficacy and side effects of antimalarials in systemic lupus erythematosus: a systematic review. Ann Rheum Dis. 2010;69(1):20-8. doi: 10.1136/ard.2008.101766. [PubMed: 19103632].

32. Nagaratnam N, Chetiyawardana AD, Rajiyah S. Aplasia and leukaemia following chloroquine therapy. Postgrad Med J. 1978;54(628):108-12. DOI: 10.1136/pgmj.54.628.108. [PubMed: 273209].

33. Abu-Shakra M, Lee P. Hypoglycemia: an unusual adverse reaction to chloroquine. Clin Exp Rheumatol. 1994;12(1):95. [PubMed: 8162653].

34. Soria A, Barbaud A, Assier H, Avenel-Audran M, Tétart F, Raison-Peyron N, et al. Cutaneous adverse drug reactions with antimalarials and allergological skin tests. Dermatology. 2015;231(4):353-9. doi: 10.1159/000438787. [PubMed: 26457932].

35. Bhatia MS, Malik SC. Psychiatric complications of chloroquine. Indian J Psychiatry. 1994;36(2):85-7. [PubMed: 21743675].

36. Alisky JM, Chertkova EL, Iczkowski KA. Drug interactions and pharmacogenetic reactions are the basis for chloroquine and mefloquine-induced psychosis. Med Hypotheses. 2006; 67(5):1090-4. DOI: 10.1016/j.mehy.2006.01.059. [PubMed: $16843615]$

37. Wolfe MS, Cordero JF. Safety of chloroquine in chemosuppression of malaria during pregnancy. $\mathrm{Br}$ Med $\mathrm{J}$. 1985;290(6480):1466-7. doi: 10.1136/bmj.290.6480.1466. [PubMed: 3922534].

38. Ressel G. AAP updates statement for transfer of drugs and other chemicals into breast milk. American Academy of Pediatrics. Am Fam Physician. 2002;65(5):979-80. [PubMed: 11898968].

39. Saleh M, Gabriels J, Chang D, Kim BS, Mansoor A, Mahmood E, et al. The effect of chloroquine, hydroxychloroquine and azithromycin on the corrected QT interval in patients with SARS-CoV-2 infection. Circ Arrhythm Electrophysiol. 2020; 13(6):e008662. doi: 10.1161/CIRCEP.120.008662. [PubMed: 32347743].

40. Zheng YY, Ma YT, Zhang JY, Xie X. COVID-19 and the cardiovascular system. Nat Rev Cardiol. 2020;17(5):259-60. 
doi: 10.1038/s41569-020-0360-5. [PubMed: 32139904].

41. Giudicessi JR, Noseworthy PA, Friedman PA, Ackerman MJ. Urgent guidance for navigating and circumventing the qtcprolonging and torsadogenic potential of possible pharmacotherapies for coronavirus disease 19 (COVID-19). Mayo Clin Proc. 2020;95(6):1213-21. doi: 10.1016/j.mayocp. 2020.03.024. [PubMed: 32359771].

42. Wu CI, Postema PG, Arbelo E, Behr ER, Bezzina CR, Napolitano C, et al. SARS-CoV-2, COVID-19, and inherited arrhythmia syndromes. Heart Rhythm. 2020;17(9):1456-62. doi: 10.1016/j.hrthm.2020.03.024. [PubMed: 32244059].

43. Lakkireddy DR, Chung MK, Gopinathannair R, Patton KK,
Gluckman TJ, Turagam $M$, et al. Guidance for cardiac electrophysiology during the coronavirus (COVID-19) pandemic from the heart rhythm society COVID-19 task force; electrophysiology section of the american college of cardiology; and the electrocardiography and arrhythmias committee of the council on clinical cardiology, american heart association. Heart Rhythm. 2020;17(9):e233-41. doi: 10.1016/j.hrthm.2020.03.028. [PubMed: 32247013].

44. Furst DE. Pharmacokinetics of hydroxychloroquine and chloroquine during treatment of rheumatic diseases. Lupus. 1996;5(Suppl 1):S11-5. [PubMed: 8803904]. 